\title{
Stable Isotope Ratios of Herbs and Spices Commonly Used as Herbal Infusions in the Italian Market
}

\author{
Purna K. Khatri, Roberto Larcher, Federica Camin, Luca Ziller, Agostino Tonon, Tiziana Nardin, \\ and Luana Bontempo*
}

Cite This: ACS Omega 2021, 6, 11925-11934

Read Online

\section{ACCESS}

山ll Metrics \& More

Article Recommendations

Supporting Information

ABSTRACT: Stable isotope ratio analysis has been widely used for traceability and authenticity purposes in relation to various food commodities, but only in a limited number of herb and spice species. This study explored the stable isotope ratios of carbon, nitrogen, sulfur, oxygen, and hydrogen $\left(\delta^{13} \mathrm{C}, \delta^{15} \mathrm{~N}, \delta^{34} \mathrm{~S}, \delta^{18} \mathrm{O}\right.$, and $\delta^{2} \mathrm{H}$ ) of 119 herbs and spices belonging to 116 plant species and 57 plant families collected from the Italian market for the first time. The characteristic value ranges of $\delta^{13} \mathrm{C}, \delta^{15} \mathrm{~N}, \delta^{34} \mathrm{~S}, \delta^{18} \mathrm{O}$, and $\delta^{2} \mathrm{H}$ of the herbs and spices went from -31.0 to $-11.6,-4.7$ to 12.0 , -5.0 to $22.0,14.7$ to 46.0 , and -158 to $-12 \%$, respectively. The isotopic profiles within and between common botanical families

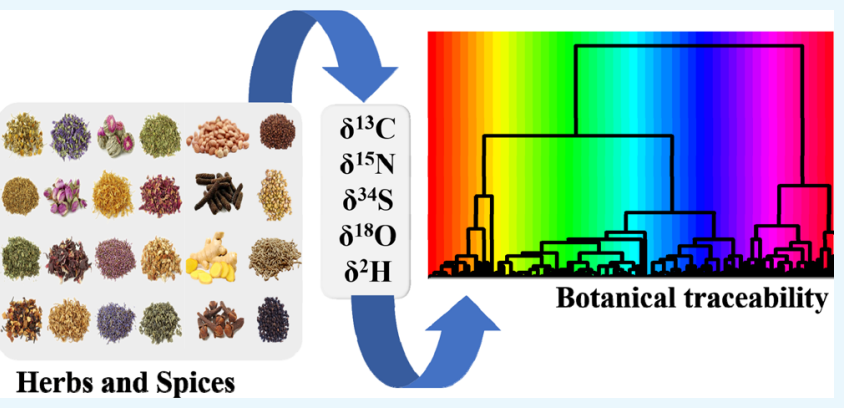
and their similarity/dissimilarity between herbs and spices belonging to the common botanical families are also discussed here. The results of this exploratory work highlight the possibility of characterizing herbs and spices and suggest widening the scope of the survey through more extensive sampling and focusing on specific plant species.

\section{INTRODUCTION}

Herbs and spices have long been used as food supplements to impart aroma, flavor, and color. They are rich sources of natural antioxidants and beneficial for human health. ${ }^{1,2}$ Many herbs and spices are also consumed as infusions, synonymously called herbal infusion/tea, or tisane. ${ }^{3}$ They are preferable to traditional tea and are coffee substitutes because they have comparably less caffeine and theine and provide a therapeutic or recreational value. Furthermore, previous studies on the clinical efficacy and safety of herbal teas have shown that the intake of herbal tea (lavender, chamomile, fenugreek, stinging nettle, spearmint, hibiscus, etc) has positive effects on female health, diabetes, heart disease, and weight loss. ${ }^{1}$ For these reasons, herbal infusions are becoming more and more popular beverages. ${ }^{3}$

The use of herbs and spices is expanding all over the world. Though the largest share of production and consumption of herbs and spices is occupied by the Asia-Pacific regions, the market in North America and Europe has also significantly increased in the last few decades. ${ }^{4,5}$ For instance, Italy has tripled the production and export of herbs and spices and doubled their import since the beginning of the millennium and is currently one of the top five producing countries in Europe and the world's first user of herbs for the cosmetic sector. It is estimated that the Italian national market of herbs and spices is worth about $\$ 750$ million in a global market that exceeds $\$ 6$ billion. $^{6}$

Generally, herbs and spices are high price commodities and are often sold as dried plant parts or in powder form. They have a long and complex supply chain. ${ }^{7}$ For this reason, they are often a target of adulteration, mislabeling, or substitution with cheaper products. For instance, a survey of 78 retail and online oregano samples in the United Kingdom resulted in $24 \%$ of oregano samples having been adulterated with olive or myrtle leaves. The amount of adulteration ranged from 30\% to over $70 \%$ of sample weight and two samples had almost no oregano leaves. ${ }^{8}$ The substitution or adulteration with one or more other plant materials could adversely affect the consumer's health because some plant species can accumulate a higher quantity of alkaloids ${ }^{9}$ or trace elements ${ }^{10}$ than other species. Thus, adulteration of herbs and spices has more than economic repercussions, as it is also a direct threat to public health due to the potentially high content of toxic phytochemicals, synthetic compounds, heavy metals, or allergens. Therefore, one of the biggest challenges for the herbs and spices supply chain is to control such frauds and economically motivated adulteration.

Received: January 15, 2021

Accepted: March 29, 2021

Published: April 28, 2021

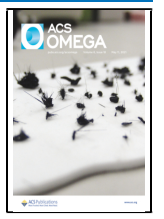

https://doi.org/10.1021/acsomega.1 1c00274 ACS Omega 2021, 6, 11925-11934 
Several studies were conducted in the past to investigate different methods to control herbs and spices fraud and adulteration. $^{11-13}$ These studies included adulteration of limited plant species such as saffron (Crocus sativus), turmeric (Curcuma domestica), black pepper (Piper nigrum), ginger (Zingiber officinale), rosemary (Salvia rosmarinus), thyme (Thymus vulgaris), oregano (Origanum vulgare), cinnamon (Cinnamomum sp.), cumin (Cuminum cyminum), sage (Salvia officinalis), and heath speedwell (Veronica officinalis). However, hundreds of herb and spice species are used for herbal infusions $s^{3,14}$ and we have limited knowledge about their traceability and authenticity. Stable isotope ratio analysis is a widely used analytical method to test the traceability and authenticity of various food commodities. ${ }^{15,16}$ Stable isotope ratios of carbon $\left(\delta^{13} \mathrm{C}\right)$, nitrogen $\left(\delta^{15} \mathrm{~N}\right)$, sulfur $\left(\delta^{34} S\right)$, oxygen $\left(\delta^{18} \mathrm{O}\right)$, and hydrogen $\left(\delta^{2} \mathrm{H}\right)$ are influenced by parameters such as climate, rainfall, physiological/metabolic pathway (i.e., photosynthesis, evapotranspiration), geographical characteristics such as altitude, latitude, coastline proximity, as well as soil properties, cultivation practices, and mycorrhizal associations. ${ }^{17-21}$ Thus, stable isotope ratios of bioelements are able to discriminate plants based on their biological and geographical origin and cultivation practices. ${ }^{22}$

This analytical approach has also been applied to a few herb and spice species to investigate the cultivation practices of chicory roots (Cichorium intybus) ${ }^{23}$ and pepper (Capsicum annuum $){ }^{24}$ geographical origin of saffron ${ }^{25,26}$ and Asian ginseng (Panax ginseng), ${ }^{19,27}$ botanical origin of vanillin, ${ }^{28} \mathrm{~N}$ fluxes of bladderwrack (Fucus vesiculosus), ${ }^{29}$ and photosynthetic pathways of various medicinal plants. ${ }^{30}$ To date, the stable isotope ratios of other herbs and spices have not been extensively explored. This study was developed to characterize herb and spice species that are commonly used for herbal infusion in the Italian market, based on the stable isotope ratio analysis of bioelements. Therefore, this paper aims to start exploring and determining the characteristic value ranges for the isotope ratios of different types of herbs and spices, focusing on those commonly available on the Italian market.

\section{RESULT AND DISCUSSIONS}

Here, we present the isotopic ranges of each element studied in 119 samples from 116 plant species and 57 botanical families and describe them from a general point of view. Then, we compare the isotopic profiles of the herbs and spices belonging to the common botanical families within and between botanical families.

2.1. General Descriptions of Stable Isotope Ratios in Herb and Spice Samples. 2.1.1. Carbon Isotope Ratios. The stable isotope ratio of carbon $\left(\delta^{13} \mathrm{C}\right)$ in all herbs and spices $(n=119)$ ranges from -31.1 to $-11.6 \%$ with the median value of $-28.0 \%$ (Table 1 ). The $\delta^{13} \mathrm{C}$ value of terrestrial plants varies primarily according to their photosynthetic pathways; the carbon assimilation in plants occurs through the Calvin cycle (i.e., $\mathrm{C}_{3}$ plants), the Hatch-Slack $\mathrm{C}$ assimilation pathway $\left(\mathrm{C}_{4}\right.$ plants), or the Crassulacean acid metabolism (CAM plants) ${ }^{22}$ with representative $\delta^{13} \mathrm{C}$ values between -35 and $-21,-14$ and -10 , and -20 to $-10 \%$, respectively. ${ }^{22}$ The $\mathrm{C}_{3}$ plants presented lower $\delta^{13} \mathrm{C}$ values due to the significant isotopic discrimination against ${ }^{13} \mathrm{CO}_{2}$ by rubisco enzyme ( $20 \%$ compared to atmospheric $\mathrm{CO}_{2}$ ) during $\mathrm{CO}_{2}$ fixation. In contrast, the isotopic discrimination against ${ }^{13} \mathrm{CO}_{2}$ by rubisco is limited in $\mathrm{C}_{4}$ plants due to the
Table 1. Summary of Stable Isotope Ratios of Carbon, Nitrogen, Sulfur, Oxygen, and Hydrogen in Herbs and Spices $(n=119)$

\begin{tabular}{lccccc} 
& $\delta^{13} \mathrm{C}(\% \circ)$ & $\delta^{15} \mathrm{~N}(\% \circ)$ & $\delta^{34} \mathrm{~S}(\% \circ)$ & $\delta^{18} \mathrm{O}(\% \circ)$ & $\delta^{2} \mathrm{H}(\% \circ)$ \\
min & -31.1 & -4.7 & -5.1 & 14.7 & -158 \\
1st Qu. & -28.7 & -0.3 & 2.7 & 21.0 & -102 \\
median & -28.0 & 2.0 & 4.5 & 22.8 & -91 \\
mean & -27.5 & 1.9 & 5.1 & 23.7 & -86 \\
3rd Qu. & -27.0 & 3.7 & 6.5 & 25.6 & -71 \\
$\max$ & -11.6 & 12.4 & 22.2 & 46.3 & -12 \\
\hline
\end{tabular}

morphological variation of bundle sheath cells. CAM plants present $\delta^{13} \mathrm{C}$ values intermediate between $\mathrm{C}_{3}$ and $\mathrm{C}_{4}$ plants, allowing for the $\mathrm{CO}_{2}$ fixation through both pathways.

In this study, the $\delta^{13} \mathrm{C}$ value of 116 herbs and spices fell between -31 and $-24 \%$ within the range of $\mathrm{C}_{3}$ plants (Table $2)$. The two plant species couch grass (Agropyron repens) and maize (Zea mays) showed a $\delta^{13} \mathrm{C}$ value -13.2 and $-11.6 \%$, respectively, representing $\mathrm{C}_{4}$ plants (Table 2 ). In addition, the $\delta^{13} \mathrm{C}$ value of a seaweed, bladderwrack $-16 \%$ (Table 2), corresponded to that of macrophytes because the source of $\mathrm{C}$ in bladderwrack is dissolved inorganic $\mathrm{C}$ with $\delta^{13} \mathrm{C}$ values around $0 \% 0$ by definition, and the $\delta^{13} \mathrm{C}$ value of such macrophytes is usually between -13 and $-20 \%$ depending upon the source and spatial variability. ${ }^{29}$

Besides the photosynthetic pathways, other factors such as stomatal conductance, environmental indicators (temperature, solar radiation, relative humidity, and rainfall), nutrient availability, and pathological stress also contribute to the plant's $\delta^{13} \mathrm{C}$ value. ${ }^{17}$ For instance, reduced photosynthesis due to a limited supply of nutrients and water induces a partial closure of stomata, which reduces the intercellular to ambient $\mathrm{CO}_{2}$ concentrations (ci/ca) and thus leads to a high plant $\delta^{13} \mathrm{C}$ value. Conversely, the higher concentration of $\mathrm{ci} / \mathrm{ca}$ dilutes heavier $\mathrm{C}$ isotopes $\left({ }^{13} \mathrm{C}\right)$ due to $\mathrm{C}$ assimilation, which results in low $\delta^{13} \mathrm{C}$ values. For example, some plant species native to coastal areas or mountainous regions such as true cinnamon (Cinnamomum zeylanicum), woodruff (Asperula odorata), boldo (Peumus boldus), java tea (Orthosiphon stamineus), mistletoe (Viscum album), clove (Syzgium aromaticum merril eugenia), bilberry (Vaccinium myrtillus), and rustyback (Ceterach officinarum) presented $\delta^{13} \mathrm{C}$ values lower than $-30 \%$ o (Table 2). On the other hand, the tropical/subtropical climate could be the possible reason of the higher $\delta^{13} \mathrm{C}$ value of psyllium (Plantago psyllium, $-24.0 \%$ ), cumin $(-24.2 \% 0)$, and bardana $\left(-24.8 \% 0\right.$ ) (Table 2). Therefore, the plant $\delta^{13} \mathrm{C}$ variation noticed in this study was expected, as these plants were possibly grown/cultivated in different regions with different environmental or agricultural regimes.

2.1.2. Nitrogen Isotope Ratios. The $\delta^{15} \mathrm{~N}$ determined in this study resulted within the range of -4.7 to $12.4 \%$ (Table 1 ). In most cases, the $\delta^{15} \mathrm{~N}$ value of a plant reflects the available sources of nitrogen, and lower $\delta^{15} \mathrm{~N}$ values (close to $0 \%$ ) correspond to the nitrogen sourced from either synthetic fertilizer inputs or symbiotic $\mathrm{N}$ fixation, while higher $\delta^{15} \mathrm{~N}$ values are due to organic fertilizer inputs. ${ }^{22}$ Furthermore, drought, stress, and distance from the sea lead to an enrichment of ${ }^{15} \mathrm{~N}$ atoms in terrestrial plants. ${ }^{31}$ Here, most of the plant species (more than $80 \%$ of samples) presented $\delta^{15} \mathrm{~N}$ values in a narrow range between -3.0 and $5.0 \%$. Only 17 species had a $\delta^{15} \mathrm{~N}$ value higher than $5 \%$, including sour orange (Citrus aurantium var bigaradia, 12.4\%o), gumweed 
Table 2. Stable Isotope Ratios of Carbon, Nitrogen, Sulfur, Oxygen, and Hydrogen of Herbs and Spices in Detail

\begin{tabular}{|c|c|c|c|c|c|c|}
\hline family/species & common name & $\delta^{15} \mathrm{~N}$ & $\delta^{13} \mathrm{C}$ & $\delta^{34} \mathrm{~S}$ & $\delta^{18} \mathrm{O}$ & $\delta^{2} \mathrm{H}$ \\
\hline \multicolumn{7}{|l|}{ Acoraceae } \\
\hline Acorus calamus & calamus & 3.5 & -27.1 & 6.0 & 25.3 & -36 \\
\hline \multicolumn{7}{|l|}{ Adoxaceae } \\
\hline Sambucus nigra & elder & 4.0 & -27.0 & 2.0 & 27.1 & -69 \\
\hline \multicolumn{7}{|l|}{ Anacardiaceae } \\
\hline Schinus molle & peruvian pepper & 3.0 & -28.1 & 13.5 & 27.1 & -76 \\
\hline \multicolumn{7}{|l|}{ Araliaceae } \\
\hline Eleutherococcus senticosus & Siberian ginseng & -0.1 & -28.2 & 5.6 & 18.4 & -116 \\
\hline Panax ginseng & ginseng & -0.2 & -27.1 & 5.9 & 21.8 & -77 \\
\hline \multicolumn{7}{|l|}{ Aspleniaceae } \\
\hline Ceterach officinarum & rustyback & -3.0 & -30.0 & 3.8 & 23.0 & -93 \\
\hline \multicolumn{7}{|l|}{ Asteraceae } \\
\hline Achillea millefolium & yarrow & -0.3 & -29.1 & 4.6 & 24.8 & -96 \\
\hline Anthemis nobilis & chamomile & 5.4 & -28.8 & 3.0 & 23.1 & -87 \\
\hline Arctium lappa & bardana & -0.5 & -24.8 & 5.2 & 23.4 & -71 \\
\hline Artemisia abrotanum & southernwood & 1.8 & -26.9 & -1.9 & 22.8 & -111 \\
\hline Artemisia absinthium & wormwood & 2.9 & -26.9 & 8.6 & 24.7 & -98 \\
\hline Calendula officinalis & marigold & 2.3 & -29.3 & 4.5 & 30.9 & -61 \\
\hline Cichorium intybus & chicory & 0.8 & -28.4 & 1.4 & 22.7 & -94 \\
\hline Cynara scolymus & Mexican arnica & 4.5 & -29.3 & 3.6 & 22.5 & -99 \\
\hline Grindelia robusta & gumweed & 8.4 & -29.9 & 3.9 & 20.6 & -98 \\
\hline Heterotheca inuloides & Mexican arnica & -0.7 & -25.6 & 1.8 & 27.9 & -91 \\
\hline Hieracium pilosella & hawkweed & -1.0 & -28.2 & 4.6 & 19.4 & -92 \\
\hline Silybum marianum & milk thistle & 4.5 & -29.9 & 2.1 & 19.4 & -114 \\
\hline Solidago virgaurea & Solidago & 1.0 & -27.7 & 4.4 & 23.7 & -111 \\
\hline Taraxacum officinale & dandelion & 2.2 & -27.0 & -0.9 & 24.3 & -80 \\
\hline \multicolumn{7}{|l|}{ Berberidaceae } \\
\hline Berberis vulgaris & barberry & -2.6 & -27.6 & 3.1 & 25.0 & -71 \\
\hline \multicolumn{7}{|l|}{ Betulaceae } \\
\hline Betula pendula & silver birch & -0.9 & -28.4 & 4.0 & 22.4 & -137 \\
\hline \multicolumn{7}{|l|}{ Bignoniaceae } \\
\hline Tabebuia avellanedae & pink trumpet tree & 5.4 & -26.4 & 7.2 & 22.5 & -52 \\
\hline \multicolumn{7}{|l|}{ Boraginaceae } \\
\hline Symphytum officinale & comfrey & 1.2 & -28.5 & -5.1 & 21.6 & -77 \\
\hline \multicolumn{7}{|l|}{ Burseraceae } \\
\hline Boswellia serrata & Indian frankincense & 0.4 & -27.4 & 8.8 & 14.7 & -158 \\
\hline \multicolumn{7}{|l|}{ Cannabaceae } \\
\hline Humulus lupulus & common hop & 4.7 & -26.3 & 3.4 & 19.3 & -87 \\
\hline \multicolumn{7}{|l|}{ Caprifoliaceae } \\
\hline Valeriana officinalis & valerian & 2.4 & -26.0 & 1.1 & 21.7 & -81 \\
\hline \multicolumn{7}{|l|}{ Crassulaceae } \\
\hline Rhodiola rosea & roseroot & -2.8 & -28.1 & 2.8 & 18.9 & -99 \\
\hline \multicolumn{7}{|l|}{ Cruciferae } \\
\hline Brassica alba & white mustard & 4.9 & -27.8 & 5.7 & 25.7 & -130 \\
\hline Capsella bursa-pastoris & Shephard's purse & 3.5 & -27.6 & 4.4 & 24.4 & -103 \\
\hline Sisymbrium officinale & hedge mustard & 2.6 & -28.0 & 6.9 & 26.4 & -83 \\
\hline \multicolumn{7}{|l|}{ Equisetaceae } \\
\hline Equisetum arvense & field horsetail & 4.5 & -27.6 & 2.5 & 21.7 & -114 \\
\hline \multicolumn{7}{|l|}{ Ericaceae } \\
\hline Arctostaphylos pungens & European blueberry & -0.7 & -27.4 & 2.2 & 27.9 & -117 \\
\hline Vaccinium myrtillus & bilberry manzanita & -4.7 & -30.1 & 5.3 & 26.8 & -86 \\
\hline
\end{tabular}


Table 2 . continued

\begin{tabular}{|c|c|c|c|c|c|c|}
\hline family/species & common name & $\delta^{15} \mathrm{~N}$ & $\delta^{13} \mathrm{C}$ & $\delta^{34} S$ & $\delta^{18} \mathrm{O}$ & $\delta^{2} \mathrm{H}$ \\
\hline $\begin{array}{l}\text { Fagaceae } \\
\text { Quercus petraea } \\
\text { Fucaceae }\end{array}$ & sessile oak & -2.1 & -26.7 & 2.7 & 14.7 & -98 \\
\hline $\begin{array}{l}\text { Fucus vesiculosus } \\
\text { Getianaceae }\end{array}$ & rockweed & 7.2 & -16.1 & 22.2 & 21.6 & -93 \\
\hline $\begin{array}{l}\text { Gentiana lutea } \\
\text { Ginkoaceae }\end{array}$ & genziana & -0.5 & -26.3 & 6.7 & 22.3 & -94 \\
\hline $\begin{array}{l}\text { Ginko biloba } \\
\text { Graminacee }\end{array}$ & ginkgo & 2.0 & -28.9 & 3.0 & 20.1 & -102 \\
\hline Agropyron repens & couch grass & -4.2 & -13.2 & 2.1 & 28.9 & -66 \\
\hline $\begin{array}{l}\text { Zea mays } \\
\text { Hypericaceae }\end{array}$ & maize & 0.3 & -11.6 & 4.6 & 20.7 & -64 \\
\hline $\begin{array}{l}\text { Hypericum perforatum } \\
\text { Juglandaceae }\end{array}$ & St. Jonh's-wort & -1.2 & -29.3 & 5.9 & 23.1 & -114 \\
\hline $\begin{array}{l}\text { Juglans sps. } \\
\text { Lamiaceae }\end{array}$ & walnut & 1.3 & -27.1 & 6.2 & 26.7 & -46 \\
\hline Hyssopus officinalis & hyssop & -1.0 & -27.2 & 2.4 & 23.3 & -106 \\
\hline Lavandula hybrid & lavender & 1.0 & -28.6 & 4.7 & 26.0 & -93 \\
\hline Melissa officinalis & balm mint & 3.4 & -28.3 & 4.5 & 20.7 & -112 \\
\hline Mentha piperita & peppermint & 5.3 & -27.3 & 4.2 & 20.9 & -113 \\
\hline Ocymum basilicum & basil & 5.5 & -28.3 & 7.9 & 27.1 & -59 \\
\hline Origanum majorana & marjoram & 1.6 & -29.0 & 5.7 & 29.2 & -56 \\
\hline Origanum vulgare & oregano & 4.0 & -27.4 & 7.9 & 22.7 & -120 \\
\hline Orthosiphon stamineus & java tea 1 & 1.1 & -29.8 & 4.2 & 20.2 & -90 \\
\hline Orthosiphon stamineus & java tea 2 & 1.3 & -30.4 & 3.9 & 18.0 & -101 \\
\hline Thymus serphyllum & wild thyme & 0.4 & -29.1 & 4.2 & 21.8 & -108 \\
\hline $\begin{array}{l}\text { Salvia officinalis } \\
\text { Lauraceae }\end{array}$ & sage & -1.4 & -28.7 & 6.2 & 21.4 & -106 \\
\hline $\begin{array}{l}\text { Cinammomum zeylanicum } \\
\text { Leguminosae }\end{array}$ & true cinnamon & -0.6 & -31.1 & 19.6 & 21.9 & -35 \\
\hline Cassia angustifolia & Alexandria senna & 2.1 & -27.2 & 7.6 & 35.0 & -92 \\
\hline Galega officinalis & galego & -1.0 & -27.0 & -4.4 & 23.0 & -112 \\
\hline Glycyrrhiza glabra & liquorice & 0.3 & -26.1 & -3.0 & 22.8 & -74 \\
\hline Ononis spinosa & spiny restharrow & 0.4 & -28.3 & 0.8 & 23.7 & -89 \\
\hline $\begin{array}{l}\text { Trigonella foenum graecum } \\
\text { Liliaceae }\end{array}$ & fenugreek & 1.2 & -25.8 & 12.8 & 34.3 & -72 \\
\hline Ruscus aculeatus & butcher's broom & -3.3 & -27.4 & 1.6 & 28.3 & -53 \\
\hline $\begin{array}{l}\text { Smilax medica } \\
\text { Loranthaceae }\end{array}$ & common salix & -1.0 & -25.8 & 0.5 & 26.0 & -46 \\
\hline $\begin{array}{l}\text { Viscum album } \\
\text { Malvaceae }\end{array}$ & mistletoe & 2.4 & -30.2 & 1.7 & 19.5 & -75 \\
\hline Althaea officinalis & marsh mallow & 3.8 & -29.4 & 3.2 & 19.3 & -115 \\
\hline $\begin{array}{l}\text { Malva sylvestris } \\
\text { Monimiaceae }\end{array}$ & cheeses & 6.6 & -28.9 & 9.3 & 24.3 & -95 \\
\hline $\begin{array}{l}\text { Peumus boldus } \\
\text { Myristicaceae }\end{array}$ & boldo & -1.3 & -30.5 & 10 & 30.9 & -71 \\
\hline $\begin{array}{l}\text { Myristica fragrans } \\
\text { Myrtaceae }\end{array}$ & nutmeg & 3.6 & -27.3 & 16.2 & 23.2 & -79 \\
\hline Pimenta officinalis & allspice & 1.5 & -28.2 & 3.7 & 23.8 & -61 \\
\hline $\begin{array}{l}\text { Syzygium aromaticum } \\
\text { Oleaceae }\end{array}$ & clove & 2.1 & -30.4 & 18 & 25.4 & -37 \\
\hline $\begin{array}{l}\text { Olea europaea } \\
\text { Onaeraceae }\end{array}$ & olive & 3.5 & -27.1 & 1.5 & 23.7 & -91 \\
\hline $\begin{array}{l}\text { Epilobium parviflorum } \\
\text { Papaveraceae }\end{array}$ & hoary willow herb & 6.5 & -27.0 & 0.2 & 21.1 & -107 \\
\hline Chelidonium majus & greater celidona & 5.7 & -28.3 & 4.8 & 21.4 & -101 \\
\hline Fumaria officinalis & fumitory & 3.2 & -26.1 & 7.1 & 24.7 & -95 \\
\hline
\end{tabular}


Table 2 . continued

\begin{tabular}{|c|c|c|c|c|c|c|}
\hline family/species & common name & $\delta^{15} \mathrm{~N}$ & $\delta^{13} \mathrm{C}$ & $\delta^{34} S$ & $\delta^{18} \mathrm{O}$ & $\delta^{2} \mathrm{H}$ \\
\hline \multicolumn{7}{|l|}{ Papaveraceae } \\
\hline $\begin{array}{l}\text { Eschscholtzia californica } \\
\text { Passifloraceae }\end{array}$ & \multicolumn{5}{|c|}{ Passifloraceae } & -87 \\
\hline \multicolumn{7}{|l|}{ Pedaliaceae } \\
\hline $\begin{array}{l}\text { Harpagophytum procumbens } \\
\text { Piperaceae }\end{array}$ & \multicolumn{5}{|c|}{ Piperaceae } & -73 \\
\hline \multicolumn{6}{|l|}{ Plantaginaceae } & -26 \\
\hline Plantago lanceolata & ribwort plantain & 3.1 & -29.5 & 4.7 & 19.2 & -113 \\
\hline Plantago psyllium & Psyllium & 4.2 & -24.0 & 0.4 & 33.1 & -65 \\
\hline Veronica officinalis & heath speedwell 1 & -0.1 & -28.9 & 4.0 & 22.4 & -101 \\
\hline \multicolumn{6}{|l|}{ Polygonaceae } & -107 \\
\hline Rumex crispus & curly dock & 4.0 & -26.8 & 7.2 & 23.8 & -66 \\
\hline Polygonum aviculare & common knotgrass & 6.5 & -28.7 & 2.6 & 22.3 & -97 \\
\hline \multicolumn{6}{|l|}{ Rhamnaceae } & -87 \\
\hline \multicolumn{6}{|l|}{ Rosaceae } & -83 \\
\hline Agrimonia eupatoria & agrimony & 0.8 & -28.3 & 4.9 & 19.0 & -109 \\
\hline Alchemilla vulgaris & lady's mantle & -1.2 & -27.4 & 6.3 & 20.8 & -103 \\
\hline Crataegus oxyacantha & hawthorns & -2.3 & -28.6 & 3.2 & 22.7 & -93 \\
\hline Filipendula ulmaria max & meadowsweet & 3.2 & -28.5 & 4.5 & 20.0 & -108 \\
\hline Prunus cerasus & sour cherry & 2.0 & -26.8 & 3.3 & 24.4 & -66 \\
\hline \multicolumn{6}{|l|}{ Rubiaceae } & -99 \\
\hline Asperula odorata & woodruff & -4.5 & -31.1 & 3.9 & 21.2 & -90 \\
\hline Cinchona succirubra & cinchona & -2.7 & -28.2 & 3.3 & 17.0 & -78 \\
\hline \multicolumn{6}{|l|}{ Rutaceae } & -94 \\
\hline Citrus aurantium var bigaradia & bitter orange & 12.4 & -26.4 & 11.7 & 34.6 & -16 \\
\hline \multicolumn{6}{|l|}{ Salicaceae } & -98 \\
\hline Salix alba & white willow 1 & 2.7 & -29.3 & 1.4 & 16.7 & -102 \\
\hline \multicolumn{6}{|l|}{ Sapindaceae } & -91 \\
\hline $\begin{array}{l}\text { Paullina cupana } \\
\text { Schisandraceae }\end{array}$ & guarana & 5.0 & -27.3 & 13.9 & 27.3 & -18 \\
\hline $\begin{array}{l}\text { Schisandra chinensis } \\
\text { Scrophulariaceae }\end{array}$ & five-flavor berry & -3.3 & -27.7 & 6.0 & 23.7 & -85 \\
\hline \multicolumn{6}{|l|}{ Theaceae } & -104 \\
\hline $\begin{array}{l}\text { Thea sinensis } \\
\text { Ulmaceae }\end{array}$ & \multicolumn{5}{|c|}{ Ulmaceae } & -70 \\
\hline Ulmus campestris & field elm & 1.3 & -27.1 & 5.5 & 15.2 & -77 \\
\hline \multicolumn{7}{|l|}{ Umbelliferae } \\
\hline Anethum graveolens & dill & 3.3 & -27.5 & 0.2 & 30.0 & -89 \\
\hline Angelica archangelica & angelica & 3.5 & -25.2 & -1.4 & 22.7 & -60 \\
\hline Carum carvi & caraway & 2.9 & -25.3 & 13.4 & 46.3 & -51 \\
\hline Coriandrum sativum & coriander & 2.0 & -28.4 & 4.6 & 23.6 & -111 \\
\hline Cuminum cyminum & cumin & 1.9 & -24.2 & 6.3 & 37.9 & -98 \\
\hline Daucus carota & wild carrot & 0.6 & -28.1 & 6.0 & 24.7 & -106 \\
\hline Hydrocotyle asiatica & pennywort & 3.3 & -28.3 & 14.4 & 18.8 & -106 \\
\hline Petroselinum crispum & parsley & 7.6 & -28.2 & 6.8 & 26.6 & -73 \\
\hline Pimpinella anisum & anise & 3.3 & -27.3 & 10.5 & 35.0 & -62 \\
\hline \multicolumn{7}{|l|}{ Urticaceae } \\
\hline Parietaria officinalis & litchwort & -2.9 & -29.4 & 4.5 & 19.1 & -97 \\
\hline Urtica dioica & nettles & 6.7 & -27.6 & 3.1 & 19.1 & -100 \\
\hline
\end{tabular}


Table 2. continued

\begin{tabular}{|c|c|c|c|c|c|c|}
\hline family/species & common name & $\delta^{15} \mathrm{~N}$ & $\delta^{13} \mathrm{C}$ & $\delta^{34} S$ & $\delta^{18} \mathrm{O}$ & $\delta^{2} \mathrm{H}$ \\
\hline \multicolumn{7}{|l|}{ Verbenaceae } \\
\hline Lippia citriodora & lemon verbena & 7.1 & -28.3 & 8.4 & 24.6 & -93 \\
\hline \multicolumn{7}{|l|}{ Violaceae } \\
\hline Viola tricolor & wild pansy & 2.3 & -28.1 & 4.2 & 23.9 & -100 \\
\hline \multicolumn{7}{|l|}{ Zingiberaceae } \\
\hline Aframomum melegueta & grains of paradise & 4.9 & -28.4 & 11.4 & 26.7 & -35 \\
\hline Elettaria cardamomum & green cardamom & 1.1 & -27.7 & 8.4 & 26.4 & -12 \\
\hline Zingiber officinale & ginger & 2.3 & -26.6 & 1.0 & 21.5 & -83 \\
\hline
\end{tabular}

(Grindelia robusta, 8.4\%o), parsley (Petroselinum crispum, $7.6 \%$ ), stickywilly (Galium aparine, $7.2 \%$ ), bladderwrack (7.2\%o), lemon verbena (Lippia citriodora, $7 \%$ ), nettle (Urtica dioica, 6.7\%o), cheeses (Malva sylvestris, 6.6\%o), hoary willowherb (Epilobium parviflorum, 6.5\%o), and so forth (Table 2). It seems that these plant species derive $\mathrm{N}$ from organic fertilizer inputs because $\mathrm{N}$ volatilization during the $\mathrm{N}$ mineralization process enhances heavy nitrogen isotope $\left({ }^{15} \mathrm{~N}\right)$ enrichment in the $\mathrm{N}$ sources available for plants and results in a high plant $\delta^{15} \mathrm{~N}$ value. ${ }^{32}$ Another possible explanation for higher $\delta^{15} \mathrm{~N}$ values could be that these plant species grew closer to sea or in an arid climate. ${ }^{29,31}$ For instance, gumweed and bladderwrack are species native to coastal areas, and the $\delta^{15} \mathrm{~N}$ values of such plants correspond to the sources of $\mathrm{N}$ (i.e., agricultural $\mathrm{N}$ or sewage waste water) ${ }^{29}$ or sea spray (oceanic nitrate). ${ }^{31}$ Besides, some plant species such as field horsetail (Equisetum arvense) and gentian (Gentiana lutea) enriched their ${ }^{13} \mathrm{C}$ and ${ }^{15} \mathrm{~N}$ isotopes content as compared to adjacent plant species due to the fully or partial mycorrhizal association, respectively. ${ }^{33,34}$

On the other hand, six plant species had $\delta^{15} \mathrm{~N}$ values lower than $-3.0 \%$ such as bilberry $(-4.7 \% 0)$, woodruff $(-4.5 \% 0)$, couch grass $(-4.2 \%$ ) , red raspberry (Rubus idaeus, $-3.7 \%$ ), Mongolia berry (Schisandra chinensis, $-3.3 \%$ ), and butcher's broom (Ruscus aculeatus, -3.3\%o) (Table 2). Besides, other factors, such as dry atmospheric depositions, physiology and metabolism (uptake, transport, and acquisition), cultivation regime and so forth, may also influence the plant's $\delta^{15} \mathrm{~N}$ value. ${ }^{19,20}$ The slightly negative $\delta^{15} \mathrm{~N}$ values of these species might be the result of substantial isotopic fractionations during plant physiology and metabolism (uptake, transport, and acquisition $)^{20}$ if an excess of $\mathrm{N}$ is available to plants or plants receive NOx pollutants that are depleted in ${ }^{15} \mathrm{~N}$ content as sources of $\mathrm{N}$.

2.1.3. Sulfur Isotope Ratios. The sulfur isotope ratio $\left(\delta^{34} S\right)$ of the plant species was between -5.1 and $22.2 \%$ (Table 1 ). Because of the reduction in atmospheric pollution, a major source of $S$ for plants is soil $S$ or fertilizer $S$ inputs and the $\delta^{34} S$ of soil and fertilizers is then reflected in the plants. ${ }^{35}$ No significant isotopic fractionation occurs during plant uptake and metabolism, ${ }^{18}$ so most of the cultivated plant species should present a $\delta^{34} \mathrm{~S}$ value close to that of the soil or fertilizer. The soil $\delta^{34} \mathrm{~S}$ varies largely with the sources; the range is between -50 and $40 \%$ from sedimentary rock to seawater sulfate, ${ }^{36}$ whereas the $\delta^{34} \mathrm{~S}$ value of the most common fertilizer is between -10 and $10 \%$ o. ${ }^{37}$ On the other hand, the oxidation of $S$ minerals (i.e., pyrite) results in a low $\delta^{34} S$ value. ${ }^{18}$

Here, most of the plant species had a $\delta^{34} S$ value of less than $10 \%$. It seems that most of the herbs in the present study get their $S$ from fertilizers or the oxidation of mineral soils. For instance, comfrey (Symphitum officinale, $-5.1 \%$ ), galego (Galega officinalis, $-4.4 \%$ ), and liquorice (Glycyrrhiza glabra, $-3.0 \%$ ) are species native to river basins or natural grasslands (Table 2). Only 13 plant species exceeded $\delta^{34} \mathrm{~S}$ values of $10 \%$ (Table 2). The highest $\delta^{34} S$ values were observed in plant species native to the coastal areas such as bladderwrack $(22.2 \% 0)$, true cinnamon (19.6\%o), clove (18\%o), and nutmeg (Myristica fragrans, 16.2\%o) (Table 2) due to the so called "sea spray effect".

2.1.4. Oxygen Isotope Ratios. The oxygen isotope ratios $\left(\delta^{18} \mathrm{O}\right)$ of the herb samples were in the range of 14.7 to $46 \%$, with median values of about $23 \%$ (Table 1 ). The $\delta^{18} \mathrm{O}$ of the plant organic matter is the sum of the isotopic signature of source water, its isotopic enrichment through transpiration pool (leaf water), and the isotope exchange between leaf water and source water, $\mathrm{CO}_{2}$, and biomolecules. ${ }^{21,39,40}$ The isotopic signature of source water (e.g., precipitation) is influenced by climatic (i.e., temperature and relative humidity) and geographical (i.e., proximity to evaporative sites and altitude) factors and as there is no isotopic fractionation during water uptake, the $\delta^{18} \mathrm{O}$ of leaf water reflects the geographical origin of the plant, even in the presence of evapotranspiration processes. In general, a plant's $\delta^{18} \mathrm{O}$ value is about $28 \%$ o higher than the $\delta^{18} \mathrm{O}$ value of source water. ${ }^{21}$ The plant's highest $\delta^{18} \mathrm{O}$ values correspond to plants from tropical climates and/or close to coastal areas, whereas the low values represent plants from temperate climates or those grown at higher altitudes.

In the present study, 76 plant species presented $\delta^{18} \mathrm{O}$ values of less than $25 \%$ o (Table 2). The lowest values of $\delta^{18} \mathrm{O}$ among all herbs have been observed in herb species native to temperate regions such as sessile oak (Quercus petraea, $14.7 \%$ ), field elm (Ulmus campestris, 15.2\%o), white willow (Salix alba, 16.7\%o), cinchona (Cinchona succirubra, 17.0\%o), and mountainous species such as Indian frankincense (Boswellia serrata, 14.7\%o) and Siberian ginseng (Eleutherococcus senticosus, $18.7 \%$ ) (Table 2 ). On the other hand, nine species showed $\delta^{18} \mathrm{O}$ values of more than $30 \%$, mostly native species of tropical or subtropical climate such as caraway (Carum carvi, $46 \%$ ), cumin (37\%o), Alexandra senna (Cassia angustifolia, 35.0\%o), aniseed (Pimpinella anisum, 35.0\%o), sour orange $(34.6 \%)$ ), fenugreek (Trigonella foenum graecum, $34.3 \%$ ), psyllium (33.0\%o), pot marigold (Calendula officinalis, 30.9\%o), and boldo (30.9\%o) (Table 2).

2.1.5. Hydrogen Isotope Ratios. As expected, $\delta^{2} \mathrm{H}$ values varied from -158 to $-12 \%$ on a wider range than that of $\delta^{18} \mathrm{O}$, with most plant species having $\delta^{2} \mathrm{H}$ values lower than $-71 \%$ (Table 1 ). Similar to $\delta^{18} \mathrm{O}$, the plant $\delta^{2} \mathrm{H}$ is affected by source water and isotopic enrichment through the transpiration pool. ${ }^{41}$ Unlike $\delta^{18} \mathrm{O}$, the $\delta^{2} \mathrm{H}$ value is also influenced by various kinetic isotope fractionations during different bio- 

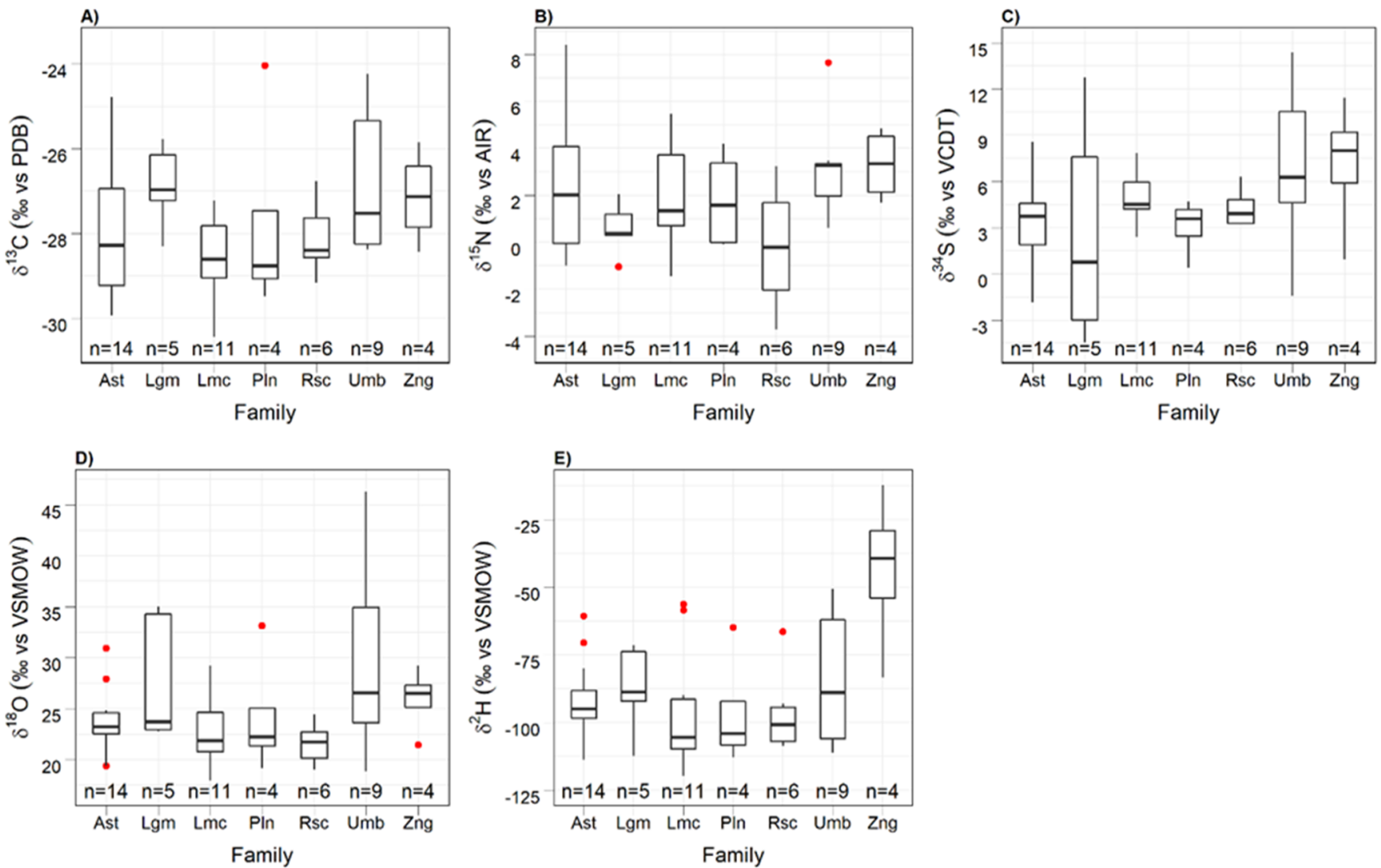

Figure 1. Comparison of stable isotope ratios of bioelements; (A) carbon, (B) nitrogen, (C) sulfur, (D) oxygen, and (E) hydrogen among common herbs/spices families. Abbreviate labels of the families; Ast $=$ Asteraceae, Lgm $=$ Leguminosae, $\mathrm{Lmc}=$ Lamiaceae, Pln $=$ Plantagoniaceae, Rsc $=$ Rosaceae, $\mathrm{Umb}=$ Umbelliferae, and Zng = Zingiberaceae. Boxes in the graph indicate interquartile ranges, horizontal lines inside the boxes are medians, and red dots are outliers. The letter " $n$ " corresponds to the number of samples.

synthetic pathways and isotope exchange with biomolecules, ${ }^{42}$ which is supported by the larger variations in the $\delta^{2} \mathrm{H}$ value as compared to the $\delta^{18} \mathrm{O}$ (Table 1). In the present study, the $\delta^{2} \mathrm{H}$ values of most plant species lie between -60 and $-130 \%$ o (Table 2). Eleven plant species which are native to tropical/ coastal climate, such as green cardamom (Elettaria cardamomum, $-12 \% 0$ ), sour orange (C. aurantium var bigaradia, $-16 \% 0$ ), guarana (Paullinia cupana, $-18 \%$ and black pepper (P. nigrum, $-26 \%$ ), grains of paradise (Aframomum melegueta, $-35 \%$ ), cinnamon (Cinammomum zeylanicum, -35\%o), calamous (Acorus calamus, $-36 \% 0)$, clove $(-37 \% 0)$, turmeric (Curcuma domestica, -44\%o), common salix (Smilax medica, $-46 \%$ ), and walnut (Juglans sp., -46\%o) (Table 2) showed the highest $\delta^{2} \mathrm{H}$ values.

On the other hand, the resin of Indian frankincense presented the lowest $\delta^{2} \mathrm{H}-158 \%$ (Table 2). Resins are constituted by terpenoids and phenolic compounds, and due to their biosynthetic pathway, it was already determined that terpenoids undergo ${ }^{13} \mathrm{C}$ depletion in the bulk plant tissues. ${ }^{43,44}$ We can suppose that the same occurs also with $\delta^{2} \mathrm{H}$. Furthermore, the low $\delta^{2} \mathrm{H}$ value of Indian frankincense can be expected due to the altitude effect, as it originates in the dry mountainous regions of India, Northern Africa, and the Middle East. ${ }^{45}$ Other two low values of $\delta^{2} \mathrm{H},-130$ and $-137 \%$, were observed in plant species native to temperate climates such as white mustard (Brassica alba) and silver birch (Betula pendula), respectively (Table 2 ).

2.2. Common Botanical Families and Herbs/Spices Classification. Figure 1A-E illustrates the stable isotope ratio profiles of common botanical families (Asteraceae, Leguminosae, Lamiaceae, Plantaginaceae, Rosaceae, Umbelliferae, and Zingiberaceae). The common botanical families had a narrow interquartile range of the $\delta^{13} \mathrm{C}$ value; a difference of less than $1.5 \%$ was observed between the third and first quartiles, except in Leguminosae and Umbelliferae specimens (Figure 1A). The $\delta^{15} \mathrm{~N}$ value close to $0 \%$ in the Leguminosae (Figure 1B) was associated with $\mathrm{N}$ fixation. ${ }^{22}$ The median value of $\delta^{15} \mathrm{~N}$ seemed to be lower in the Rosaceae family than other families (Figure 1B). In particular, the two species red raspberry and Hawthorns produced slightly negative $\delta^{15} \mathrm{~N}$ values (Table 2). However, the difference to the other families was not statistically significant $(p<0.05)$. Moreover, Umbelliferae and Zingiberaceae tended to have slightly high $\delta^{15} \mathrm{~N}, \delta^{34} \mathrm{~S}$, and $\delta^{18} \mathrm{O}$ values, but there was no statistically significant difference among them at $95 \%$ confidence (Figure $1 \mathrm{~B}-\mathrm{D})$. Though the median $\delta^{34} \mathrm{~S}$ of leguminous herbs tended to be the lowest, the values were dispersed from -4.5 to $12 \%$ o (Figure 1C). Three families, Lamiaceae, Rosaceae, and Plantaginaceae, showed comparable values of both $\delta^{18} \mathrm{O}$ and $\delta^{2} \mathrm{H}$ (Figure 1C, D). Zingiberaceae had significantly higher $\delta^{2} \mathrm{H}$ than other families $(p<0.05)$ (Figure 1D).

Despite the variabilities in isotopic profiles among the common botanical families, it was not possible to classify herbs and spices. The impact of plant parts isotopic composition on classifying the botanical families was checked. In particular, seeds and roots generally showed slightly higher isotopic values than leaves and other plant parts, but this tendency was not statistically significant $(p<0.05)$ within each botanical family. 
A dendrogram of hierarchical clustering also provided an overview of how herbs and spices of a family are dissimilar to each other (Figure S1). It seems that herbs and spices which had seeds as plant parts formed a partially separated cluster (Figure S1). However, the hierarchical cluster was mainly influenced by $\delta^{2} \mathrm{H}$ and $\delta^{18} \mathrm{O}$ values, the high values formed a cluster at the left side and low values at the right side. The result indicated that the geographical origin of herbs and spices probably limits the possible classification of botanical families of herbs and spices based on their isotopic profiles. In addition, the plant parts can be considered as a co-factor having some influence on the isotopic variability. Further research with extensive herb and spice samples of different plant parts and with a known geographical origin is recommended.

\section{MATERIALS AND METHODS}

3.1. Sampling and Sample Preparation. One-hundred and nineteen dried herb and spice samples were purchased in 2017 from a drug/herbal store in Northern Italy. All the plant samples were commercially certified products according to the botanical origin and belonged to 116 plant species and 57 plant families. A previous study has already explored the alkaloid content of the same samples. ${ }^{9}$ The samples included different plant parts such as leaves, foliage (whole above-ground biomass), stem, bark, flower, stigma, fruits (or berries), seed, and roots. Plant samples were grounded and homogenized with a mechanical shaker (Cryomill, Retsch $\mathrm{GmbH}$, Germany).

3.2. Stable Isotope Ratio Analysis. About $0.25 \pm 0.05$ $\mathrm{mg}$ of duplicate plant samples was weighed into silver capsules for oxygen and hydrogen isotope ratio analysis and introduced into the TC-IRMS (a pyrolizer-Finnigan TC/EA, hightemperature conversion elemental analyzer, Thermo Scientific, Bremen, Germany-coupled with an isotope ratio mass spectrometer IRMS-Finnigan DELTA XP, Thermo Scientific, Bremen, Germany). Isotope values were corrected with the International standard, USGS 54 (U.S. Geological Survey, Reston, VA, USA, certified values $\delta^{2} \mathrm{H} ;-150.4 \%$, $\delta^{18} \mathrm{O}$; $+17.79 \%$ ) and USGS 56 (U.S. Geological Survey, certified values $\left.\delta^{2} \mathrm{H} ;-44 \% 0, \delta^{18} \mathrm{O} ;+27.23 \% 0\right)$. The value precisions (i.e., standard deviation) were $<5 \% 0$ and $<1 \% 0$ for the hydrogen and oxygen isotope ratio, respectively.

The sample weight for $\mathrm{C}, \mathrm{N}$, and $\mathrm{S}$ isotope ratio analysis was calculated based on the test results. For the sample weight test, $\sim 2.5 \mathrm{mg}$ of triplicate plant samples of different plant parts (leaves, foliage, flower, bark, and root) were weighed in a tin capsule and introduced into an EA-IRMS (elemental analyzer-VARIO CUBE, Elementar Analysensysteme $\mathrm{GmbH}$, Germany-coupled with an IRMS - Isoprime, Isoprime Ltd., UK). About 2-4 mg triplicate plant samples were introduced into an EA-IRMS for the CNS isotope ratio analysis. The isotopic values for $\delta^{13} \mathrm{C}, \delta^{15} \mathrm{~N}$, and $\delta^{34} \mathrm{~S}$ were calculated against in-house working standards, which were themselves calibrated against international reference materials: potassium nitrate IAEA-NO3 (IAEA-International Atomic Energy Agency, Vienna, Austria) for $\delta^{15} \mathrm{~N}$, L-glutamic acid USGS 40 (U.S. Geological Survey, Reston, VA, USA) for ${ }^{13} \mathrm{C} /{ }^{12} \mathrm{C}$ and ${ }^{15} \mathrm{~N} /{ }^{14} \mathrm{~N}$, fuel oil NBS-22, IAEA-CH-6 for $13 \mathrm{C} /$ 12C, barium sulfates IAEASO-5, NBS 127 (IAEA), and USGS 42 and USGS 43 for $\delta^{34} \mathrm{~S}$. Through the development of a linear equation, the values were calculated against two working standards. For the $\delta^{13} \mathrm{C}, \delta^{15} \mathrm{~N}, \delta^{34} \mathrm{~S}, \delta^{18} \mathrm{O}$, and $\delta^{2} \mathrm{H}$, the uncertainty of measurement ( $1 \mathrm{Std} \mathrm{Dev})$ was $0.1,0.2,0.3,0.3$, and $1 \%$, respectively.

3.3. Data Analysis. Due to the limited information on the sample labeling and the presence of only a few samples of herb and spice species in some families, sample sizes were narrowed down to common botanical families (at least four samples in each family) for a statistical approach. Normal distribution and homogeneity of variances for each isotopic ratio were tested in RStudio software version 1.3.1073. One-way analysis of variance for normally distributed data (i.e., the $\delta^{13} \mathrm{C}, \delta^{15} \mathrm{~N}$, and $\delta^{34} \mathrm{~S}$ ) and a nonparametric test (the Kruskal-Wallis test) for the data without normal distribution $\left(\delta^{18} \mathrm{O}\right.$ and $\left.\delta^{2} \mathrm{H}\right)$ were applied to check significant differences according to the plant family or plant parts. Partial least square regression discriminant analysis and classification and regression tree were tested in XLSTAT Microsoft excel but produced low accuracy in the classification of botanical families. A dendrogram of the hierarchical clustering (Euclidean distance based on Ward's minimum variance method) was created to illustrate the dissimilarities of botanical families. All the graphics were prepared in RStudio software version 1.3.1073.

\section{ASSOCIATED CONTENT}

\section{Supporting Information}

The Supporting Information is available free of charge at https://pubs.acs.org/doi/10.1021/acsomega.1c00274.

Dendrogram of hierarchical clustering (PDF)

\section{AUTHOR INFORMATION}

\section{Corresponding Author}

Luana Bontempo - Department of Food Quality and Nutrition, Research and Innovation Centre, Fondazione Edmund Mach (FEM), 38010 San Michele all'Adige, Italy; 다이.org/0000-0001-7583-1501;

Email: luana.bontempo@fmach.it

\section{Authors}

Purna K. Khatri - Department of Food Quality and Nutrition, Research and Innovation Centre, Fondazione Edmund Mach (FEM), 38010 San Michele all'Adige, Italy; Center Agriculture Food Environment (C3A), University of Trento, 38010 San Michele all'Adige, Trentino, Italy

Roberto Larcher - Experiment and Technological Services Department, Technology Transfer Centre, Fondazione Edmund Mach (FEM), 38010 San Michele all'Adige, Italy

Federica Camin - Department of Food Quality and Nutrition, Research and Innovation Centre, Fondazione Edmund Mach (FEM), 38010 San Michele all'Adige, Italy; Center Agriculture Food Environment (C3A), University of Trento, 38010 San Michele all'Adige, Trentino, Italy; orcid.org/ 0000-0003-0509-6745

Luca Ziller - Department of Food Quality and Nutrition, Research and Innovation Centre, Fondazione Edmund Mach (FEM), 38010 San Michele all'Adige, Italy

Agostino Tonon - Department of Food Quality and Nutrition, Research and Innovation Centre, Fondazione Edmund Mach (FEM), 38010 San Michele all'Adige, Italy

Tiziana Nardin - Experiment and Technological Services Department, Technology Transfer Centre, Fondazione Edmund Mach (FEM), 38010 San Michele all'Adige, Italy

Complete contact information is available at:

https://pubs.acs.org/10.1021/acsomega.1c00274 


\section{Notes}

The authors declare no competing financial interest.

\section{ACKNOWLEDGMENTS}

Authors thanks research colleagues and technicians at Traceability Unit-FEM for the support during this study.

\section{REFERENCES}

(1) Poswal, F. S.; Russell, G.; Mackonochie, M.; MacLennan, E.; Adukwu, E. C.; Rolfe, V. Herbal Teas and Their Health Benefits: A Scoping Review. Plant Foods Hum. Nutr. 2019, 74, 266-276.

(2) Yashin, A.; Yashin, Y.; Xia, X.; Nemzer, B. Antioxidant Activity of Spices and Their Impact on Human Health: A Review. Antioxidants 2017, 6, 70 .

(3) Sõukand, R.; Quave, C. L.; Pieroni, A.; Pardo-de-Santayana, M.; Tardío, J.; Kalle, R.; Łuczaj, Ł.; Svanberg, I.; Kolosova, V.; AceitunoMata, L.; Menendez-Baceta, G.; Kołodziejska-Degórska, I.; Piroznikow, E.; Petkevičius, R.; Hajdari, A.; Mustafa, B. Plants Used for Making Recreational Tea in Europe: A Review Based on Specific Research Sites. J. Ethnobiol. Ethnomed. 2013, 9, 58.

(4) CBI. Which Trends Offer Opportunities on the European Spices and Herbs Market?. 2018. https://Cbi.Eu/Market-Information/ Spices-Herbs/Trends/ (accessed 2020-12-22).

(5) Nguyen, L.; Duong, L. T.; Mentreddy, R. S. The U.S. Import Demand for Spices and Herbs by Differentiated Sources. J. Appl. Res. Med. Aromat. Plants 2019, 12, 13-20.

(6) Vesentini, I. Spezie e Piante Officinali, Produzione Triplicata in 20 Anni: Nasce Una Fiera Ad Hoc; Il Sole 24 Ore, 2020.

(7) Guidance on Authenticity of Herbs and Spices-Industry Best Practice on Assessing and Protecting Culinary Dried Herbs and Spices; Food and Drink Federation, 2016.

(8) Black, C.; Haughey, S. A.; Chevallier, O. P.; Galvin-King, P.; Elliott, C. T. A Comprehensive Strategy to Detect the Fraudulent Adulteration of Herbs: The Oregano Approach. Food Chem. 2016, 210, 551-557.

(9) Nardin, T.; Piasentier, E.; Barnaba, C.; Larcher, R. Alkaloid Profiling of Herbal Drugs Using High Resolution Mass Spectrometry. Drug Test. Anal. 2018, 10, 423-448.

(10) Potortì, A. G.; Bua, G. D.; Lo Turco, V.; Ben Tekaya, A.; Beltifa, A.; Ben Mansour, H.; Dugo, G.; Di Bella, G. Major, Minor and Trace Element Concentrations in Spices and Aromatic Herbs from Sicily (Italy) and Mahdia (Tunisia) by ICP-MS and Multivariate Analysis. Food Chem. 2020, 313, 126094.

(11) Galvin-King, P.; Haughey, S. A.; Elliott, C. T. Herb and Spice Fraud; the Drivers, Challenges and Detection. Food Contr. 2018, 88, $85-97$.

(12) Oliveira, M. M.; Cruz-Tirado, J. P.; Barbin, D. F. Nontargeted Analytical Methods as a Powerful Tool for the Authentication of Spices and Herbs: A Review. Compr. Rev. Food Sci. Food Saf. 2019, 18, 670-689.

(13) Reinholds, I.; Bartkevics, V.; Silvis, I. C. J.; van Ruth, S. M.; Esslinger, S. Analytical Techniques Combined with Chemometrics for Authentication and Determination of Contaminants in Condiments: A Review. J. Food Compos. Anal. 2015, 44, 56-72.

(14) Tea and Herbal Infusions Europe. Inventory List of Herbals Considered as Food, 2019. https://doi.org/www.thie-online.eu.

(15) Laursen, K. H.; Bontempo, L.; Camin, F.; Roßmann, A. Advances in Isotopic Analysis for Food Authenticity Testing. Adv. Food Authenticity Test. 2016, 227-252.

(16) Camin, F.; Boner, M.; Bontempo, L.; Fauhl-Hassek, C.; Kelly, S. D.; Riedl, J.; Rossmann, A. Stable Isotope Techniques for Verifying the Declared Geographical Origin of Food in Legal Cases. Trends Food Sci. Technol. 2017, 61, 176-187.

(17) Cernusak, L. A.; Ubierna, N.; Winter, K.; Holtum, J. A. M.; Marshall, J. D.; Farquhar, G. D. Environmental and Physiological Determinants of Carbon Isotope Discrimination in Terrestrial Plants. New Phytol. 2013, 200, 950-965.
(18) Tcherkez, G.; Tea, I. 32S/34S Isotope Fractionation in Plant Sulphur Metabolism. New Phytol. 2013, 200, 44-53.

(19) Chung, I.-M.; Lee, T.-J.; Oh, Y.-T.; Ghimire, B. K.; Jang, I.-B.; Kim, S.-H. Ginseng Authenticity Testing by Measuring Carbon, Nitrogen, and Sulfur Stable Isotope Compositions That Differ Based on Cultivation Land and Organic Fertilizer Type. J. Ginseng Res. 2017, 41, 195-200.

(20) Evans, R. D. Physiological Mechanisms Influencing Plant Nitrogen Isotope Composition. Trends Plant Sci. 2001, 6, 121-126.

(21) Barbour, M. M. Stable Oxygen Isotope Composition of Plant Tissue: A Review. Funct. Plant Biol. 2007, 34, 83-94.

(22) Ehleringer, J. R. Interpreting Stable Isotope Ratios in Plants and Plant-Based Foods. In Food Forensics; Carter, J. F., Chesson, L. A., Eds.; CRC Press: Boca Raton, FL, 2017; pp 46-62.

(23) Sinkovič, L.; Nečemer, M.; Ogrinc, N.; Žnidarčič, D.; Stopar, D.; Vidrih, R.; Meglič, V. Parameters for Discrimination between Organic and Conventional Production: A Case Study for Chicory Plants (Cichorium Intybus L.). Food Chem. Toxicol. 2020, 136, 111109.

(24) Flores, P.; Fenoll, J.; Hellín, P. The Feasibility of Using $\Delta 15 \mathrm{~N}$ and $\Delta 13 \mathrm{C}$ Values for Discriminating between Conventionally and Organically Fertilized Pepper (Capsicum Annuum L.). J. Agric. Food Chem. 2007, 55, 5740-5745.

(25) Maggi, L.; Carmona, M.; Kelly, S. D.; Marigheto, N.; Alonso, G. L. Geographical Origin Differentiation of Saffron Spice (Crocus Sativus L. Stigmas) - Preliminary Investigation Using Chemical and Multi-Element (H, C, N) Stable Isotope Analysis. Food Chem. 2011, 128, 543-548.

(26) Perini, M.; Pianezze, S.; Ziller, L.; Ferrante, M.; Ferella, F.; Nisi, S.; Foschi, M.; D'Archivio, A. A. Stable Isotope Ratio Analysis Combined with Inductively Coupled Plasma-Mass Spectrometry for Geographical Discrimination between Italian and Foreign Saffron. J. Mass Spectrom. 2020, 55, No. e4595.

(27) Chung, I.-M.; Kim, J.-K.; Lee, J.-H.; An, M.-J.; Lee, K.-J.; Park, S.-K.; Kim, J.-U.; Kim, M.-J.; Kim, S.-H. C/N/O/S Stable Isotopic and Chemometric Analyses for Determining the Geographical Origin of Panax Ginseng Cultivated in Korea. J. Ginseng Res. 2018, 42, 485495.

(28) Hansen, A.-M. S.; Fromberg, A.; Frandsen, H. L. Authenticity and Traceability of Vanilla Flavors by Analysis of Stable Isotopes of Carbon and Hydrogen. J. Agric. Food Chem. 2014, 62, 10326-10331.

(29) Winde, V.; Böttcher, M. E.; Voss, M.; Mahler, A. Bladder Wrack (Fucus Vesiculosus) as a Multi-Isotope Bio-Monitor in an Urbanized Fjord of the Western Baltic Sea. Isot. Environ. Health Stud. 2017, 53, 563-579.

(30) Marchese, J. A.; Ming, L. C.; Ducatti, C.; Tadeu Da Silva, E. Carbon Isotope Composition as a Tool for Quality Control of Herbs, Spices and Medicinal Plants. Acta Hortic. 2006, 720, 185-188.

(31) Heaton, T. H. E. The $15 \mathrm{~N} / 14 \mathrm{~N}$ Ratios of Plants in South Africa and Namibia: Relationship to Climate and Coastal/Saline Environments. Oecologia 1987, 74, 236-246.

(32) Bateman, A. S.; Kelly, S. D. Fertilizer Nitrogen Isotope Signatures. Isot. Environ. Health Stud. 2007, 43, 237-247.

(33) Giesemann, P.; Rasmussen, H. N.; Gebauer, G. Partial mycoheterotrophy is common among chlorophyllous plants with Paris-type arbuscular mycorrhiza. Ann. Bot. 2021, mcab003.

(34) Giesemann, P.; Eichenberg, D.; Stöckel, M.; Seifert, L. F.; Gomes, S. I. F.; Merckx, V. S. F. T.; Gebauer, G. Dark Septate Endophytes and Arbuscular Mycorrhizal Fungi ( Paris -Morphotype ) Affect the Stable Isotope Composition of 'Classically' NonMycorrhizal Plants. Funct. Ecol. 2020, 34, 2453-2466.

(35) Tanz, N.; Schmidt, H.-L. $\delta 34$ S-Value Measurements in Food Origin Assignments and Sulfur Isotope Fractionations in Plants and Animals. J. Agric. Food Chem. 2010, 58, 3139-3146.

(36) Chalk, P. M.; Inácio, C. T.; Chen, D. Tracing S Dynamics in Agro-Ecosystems Using 34S. Soil Biol. Biochem. 2017, 114, 295-308.

(37) Otero, N.; Soler, A.; Canals, À. Fertilizer Characterization: Isotopic Data (N, S, O, C, and Sr). Environ. Sci. Technol. 2004, 38, $3254-3262$ 
(38) Thode, H. G. Sulphur Isotopes in Nature and the Environment: An Overview. In Stable Isotopes: Natural and Anthropogenic Sulphur in the Environment; Krouse, H. R., Grinenko, V. A., Eds.; John Wiley \& Sons: Chichester, New York, Brisbane, Toronto, 1991; pp 1-26.

(39) Cernusak, L. A.; Barbour, M. M.; Arndt, S. K.; Cheesman, A. W.; English, N. B.; Feild, T. S.; Helliker, B. R.; Holloway-Phillips, M. M.; Holtum, J. A. M.; Kahmen, A.; Mcinerney, F. A.; Munksgaard, N. C.; Simonin, K. A.; Song, X.; Stuart-Williams, H.; West, J. B.; Farquhar, G. D. Stable Isotopes in Leaf Water of Terrestrial Plants. Plant Cell Environ. 2016, 39, 1087-1102.

(40) Gessler, A.; Ferrio, J. P.; Hommel, R.; Treydte, K.; Werner, R. A.; Monson, R. K. Stable Isotopes in Tree Rings: Towards a Mechanistic Understanding of Isotope Fractionation and Mixing Processes from the Leaves to the Wood. Tree Physiol. 2014, 34, 796818.

(41) Farquhar, G. D.; Cernusak, L. A.; Barnes, B. Heavy water fractionation during transpiration. Plant Physiol. 2007, 143, 11-18.

(42) Schmidt, H.-L.; Werner, R. A.; Eisenreich, W. Systematics of $2 \mathrm{H}$ Patterns in Natural Compounds and Its Importance for the Elucidation of Biosynthetic Pathways. Phytochem. Rev. 2003, 2, 6185.

(43) Stern, B.; Lampert Moore, C. D.; Heron, C.; Pollard, A. M. Bulk Stable Light Isotopic Ratios in Recent and Archaeological Resins: Towards Detecting the Transport of Resins in Antiquity? Archaeometry 2008, 50, 351-370.

(44) Murray, A. P.; Edwards, D.; Hope, J. M.; Boreham, C. J.; Booth, W. E.; Alexander, R. A.; Summons, R. E. Carbon Isotope Biogeochemistry of Plant Resins and Derived Hydrocarbons. Org. Geochem. 1998, 29, 1199-1214.

(45) Siddiqui, M. Z. Boswellia Serrata, a Potential Antiinflammatory Agent: An Overview. Indian J. Pharm. Sci. 2011, 73, 255-261. 